\title{
La Responsabilidad Social como Fundamento para la Estrategia Comunicacional de Organizaciones Innovadoras
}

\author{
Social Responsibility as a Basis for the Communicational \\ Strategy of Innovative Organizations
}

\begin{abstract}
RESUMEN
La implementación de estrategias comunicacionales fundamentadas en los principios de la responsabilidad social, es de vital importancia para las organizaciones innovadoras en su accionar competitivo. A partir de la revisión documental de varios autores y fundaciones internacionales expertas en el tema, se aprecia que las estrategias comunicacionales junto con la responsabilidad social empresarial (RSE), conforman un eje estratégico para la imagen, credibilidad y posicionamiento en el mercado de las empresas innovadoras. También, se encontró en la literatura estudiada, que la cultura organizacional juega un papel clave en la adaptabilidad a los entornos cambiantes de las sociedades y en la superación de las posibles barreras comunicacionales para el trabajo colaborativo y el aprendizaje organizacional. Como conclusión se refuerza el valor estratégico para las organizaciones innovadoras, de las comunicaciones y el compromiso social a fin de alcanzar sus objetivos empresariales.
\end{abstract}

Palabras claves: Comunicación; innovación; responsabilidad social.

\begin{abstract}
The implementation of communication strategies based on the principles of social responsibility is of vital importance for innovative organizations in their competitive actions. From the documentary review of several authors and international foundations with experience in the subject, it is appreciated that communication strategies alongside with corporate social responsibility (CSR), form a strategic axis for the image, credibility and positioning in the innovative businesses market. In addition, it was found in the literature studied that the organizational culture plays a key role in the adaptability to the changing environments of societies and in overcoming the possible communication barriers for collaborative work and organizational learning. In conclusion, the strategic value for innovative organizations, communications and social commitment is reinforced in order to achieve their business objectives.

Keywords: Communication; innovation; social responsibility.
\end{abstract}

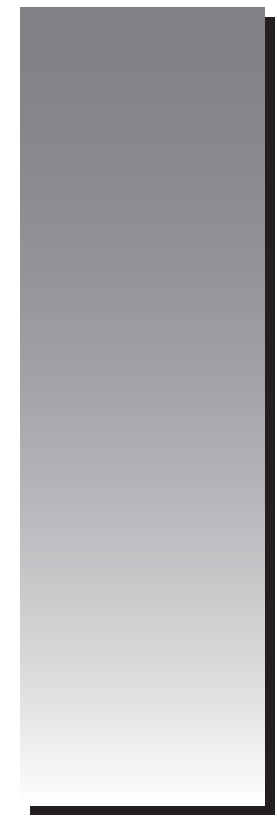

Joel José Betancourt Gamboa

joeljbg@gmail.com

Universidad Privada Dr. Rafael Belloso Chacín. Venezuela 


\section{INTRODUCCIÓN}

La responsabilidad social empresarial en el contexto actual tiene una relevancia especial como fundamento en aquellas empresas que consideran los criterios de desarrollo sostenible como parte del compromiso social para competir en los mercados.

Las empresas competitivas valoran la responsabilidad social, no solo desde el punto de vista regulatorio por parte de los organismos del estado, sino que están conscientes de los impactos de sus operaciones y productos o servicios hacia el medio ambiente y reconocen la importancia de contribuir a mitigar los problemas ambientales y sociales para el bienestar de la sociedad. Esto implica que los planes estratégicos, deben considerar estrategias que respondan al compromiso social como parte de la cultura organizacional para alcanzar las metas propuestas de productividad y competitividad, logrando a la vez, proyectar a los grupos de interés una imagen de organización socialmente responsable.

En este mismo orden de ideas, Pérez (2011) apunta que, en los últimos años se ha venido observando un entorno social dinámico que impulsa a las sociedades contemporáneas a enfrentar el reto de proyectarse, adaptarse a un proceso de cambio que ha ido avanzando hacia la construcción de nuevas sociedades, y donde el desarrollo de nuevas tendencias en el uso de las tecnologías de comunicación e información, demandan una adaptación de las organizaciones que requieren nuevas visiones e interpretaciones que les procuren aprehender este cambio.

En la actualidad, la innovación empresarial ha superado la concepción inicial de estar dirigida solo a la aplicación directa en los procesos productivos, productos y servicios, tal como lo expone, la organización, Foro Consultivo Científico y Tecnológico (FCCyT) A.C. y la Organización para la Cooperación y Desarrollo Económico (OCDE, 2012), quienes afirman:

Si bien el concepto de innovación se asocia más con productos de la tecnología y la industria, lo cierto es que debe relacionarse también con cambios estructurales que incluyan toda la cadena de valor y con modificaciones en procesos o prácticas existentes. (p.3)
Sumado a lo anterior expuesto, las organizaciones deben considerar la comunicación como una herramienta de gestión, de competitividad y de compromiso social, además de ser el pilar fundamental de las relaciones humanas. Es por ello que, en su afán de lograr una posición de liderazgo en los mercados, reconocen la importancia de poseer un sistema efectivo y eficiente de comunicación junto a una cultura de innovación con compromiso social.

\section{OBJETIVO DEL ENSAYO}

El objetivo del presente trabajo es contribuir al debate que permita profundizar en el análisis de la necesidad que tienen las organizaciones de considerar en su accionar innovador, la responsabilidad social empresarial como fundamento para el diseño e implementación de su plan estratégico comunicacional, así como para dar respuesta a las nuevas demandas de los mercados y de la sociedad en general, aportando a un crecimiento económico sostenible.

\section{ARGUMENTACIÓN}

\section{Organizaciones innovadoras y compromiso social.}

Las organizaciones innovadoras presentan características muy especiales en la forma de gerenciar el negocio, así como en la forma de abordar los problemas de índole económicos, sociales, ambientales, políticos y culturales. Concentran esfuerzos en los aspectos relacionados con la gestión del conocimiento, la aplicación de nuevas tecnologías y el desarrollo de innovadores productos, servicios y procesos que brinden mayores beneficios a sus consumidores, usuarios o clientes contribuyendo a la vez a mejorar el bienestar de la comunidad y siendo responsables con el medio ambiente y los ecosistemas.

La imagen organizacional favorece la posición competitiva cuando las organizaciones innovadoras evidencian a través de sus actividades el compromiso social. En los escenarios actuales de globalización junto a mayores facilidades de movilidad, accesibilidad y disponibilidad de comunicación instantanea, es fácil un mayor escrutinio por parte de una amplia variedad de grupos e individuos con relación a las actividades organizacionales (Lescano, Valdéz, Lescano, Reyes y Belaúnde, 2015). 
El compromiso social en las organizaciones innovadoras es un valor dentro de la cultura organizacional y es un factor clave de éxito a considerar en la formulación de las estrategias para alcanzar la visión. La implementación de soluciones novedosas a los problemas sociales y ambientales constituye parte de las ventajas competitivas, así como, el aportar al desarrollo tecnológico a través de sus unidades de ingeniería, desarrollo e innovación.

\section{Responsabilidad social y la innovación}

La responsabilidad social desde el punto de vista de su concepción, demanda a las organizaciones, la imperiosa necesidad de minimizar sus impactos negativos hacia el medio ambiente, generados por medio de sus operaciones productivas y comerciales. Además, propone que deben aportar soluciones para ayudar a mitigar los problemas ambientales que amenazan la convivencia saludable de la presente y futuras generaciones.

Ramos (2010) declara que "la responsabilidad social constituye, sin lugar a dudas, una de las manifestaciones más expresivas de la ética organizacional, al representar el compromiso de la empresa con la sociedad donde opera" (p.48). Esta declaración sugiere para una empresa socialmente responsable, la necesidad de contemplar dentro de sus planes estratégicos, políticas dirigidas a atender el crecimiento y desarrollo de sus empleados con sentido ético; aportando a la vez, a través de programas específicos, a la solución de los problemas presentados en el ámbito de la comunidad externa donde se encuentra inserta, así como de la comunidad global en materia de desarrollo sostenible y cuidado del planeta.

Dado lo critico y la complejidad actual de los problemas ambientales y sociales, la innovación surge como condición insustituible en el accionar de las empresas con miras a responder al compromiso social y para ser competitivas en los mercados, ante las nuevas exigencias. Producto de investigaciones se establece que "la innovación es un motor importante del crecimiento orgánico necesario para generar rendimientos superiores al promedio de forma sostenida" (Hitt, Ireland y Hoskisson, 2014, p.339).
Las organizaciones están inmersas en una realidad de competitividad global, lo que implica que deben innovar de manera sostenida para ser líderes del mercado. Hitt, et al. (2014) declaran:

La innovación es uno de los resultados fundamentales que las compañías persiguen con los emprendimientos y muchas veces también es fuente del éxito en la competitividad, en especial en el caso de las compañías que participan en entornos muy competidos y turbulentos. (p.402)

Es así, como las organizaciones necesitan transformar en muchos casos los sistemas de producción, diseñar nuevos procesos, y crear nuevos productos amigables con el medio ambiente; aplicando a lo largo de su cadena de valor los criterios establecidos en materia de desarrollo sostenible.

\section{Estrategia comunicacional y la responsabi- lidad social.}

Las organizaciones innovadoras, utilizan los recursos tecnológicos de información y comunicación, así como técnicas comunicacionales para el trabajo colaborativo y las redes sociales, logrando una mayor apertura hacia ambientes globales que incrementa la efectividad del flujo de información (conocimiento e innovación) para el logro de los objetivos propuestos en materia comunicacional. En línea general, "una estrategia de comunicación es esencial para la ejecución apropiada de las metas corporativas y el desarrollo y mantenimiento de la marca de una empresa y su ventaja competitiva" (Koontz, Weihrich, Cannice, 2012, p.471).

Disponer de una estrategia comunicacional efectiva constituye para las organizaciones innovadoras un factor clave de éxito dada la dinámica de los cambios en el entorno externo e interno que afectan el desempeño organizacional, siendo necesario evaluar y controlar cualquier desviación ocasionada por estos cambios. Por otro lado, cubrir los requerimientos de comunicación propios del proceso de innovación, así como, la necesidad de suministrar en tiempo real información al personal, es de vital importancia para alcanzar los resultados propuestos por la empresa. Para cumplir con estos requerimientos organizacionales es fundamental contar con un plan estratégico comunicacional como herramienta de dirección que junto con el 
plan estratégico global de la empresa aseguren una gestión exitosa.

Las organizaciones innovadoras cuentan con planes estratégicos para lograr sus objetivos de largo plazo alineados para alcanzar la visión y durante la etapa de formulación de las estrategias como en la etapa de implementación, la comunicación efectiva a lo largo de la empresa cumple una función clave para el éxito esperado. Por consiguiente, se hace necesario "emprender acciones que apoyen la estrategia, lo que significa comunicar a otros miembros de la organización la información pertinente, convencerlos de la estrategia y explicarles lo que tienen que hacer para ponerla en práctica" (Hitt, Black, y Porter, 2006, p. 491).

La estrategia comunicacional es parte de la identidad de la organización innovadora, por su función integradora, permitiendo que los conocimientos y experiencias que se generan sean transferidos al personal interno para la toma de decisiones constituyéndose en acción y a la vez, en aprendizaje dinámico organizacional. De igual manera, los contenidos seleccionados y tratados son transmitidos al público externo de interés para la empresa.

\section{El compromiso ambiental de Tesla Motors Inc. "Pionera en innovación con RSE"}

La empresa Tesla Motors Inc., desde su fundación en 2003 hasta la presente fecha ha demostrado una gran capacidad de innovación desde el diseño hasta la fabricación de sus vehículos eléctricos (VE) y en la implantación de un modelo de negocio con un sentido de compromiso social y ambiental. El enfoque de esta organización, es impulsado por una fuerte preocupación por el medio ambiente; en particular, la inminente amenaza del cambio climático global. La compañía apuesta a que los consumidores conscientes del medio ambiente y bien informados estarán cada vez más interesados en productos ecológicos. (Hitt et al., 2014)

Dado los desafíos significativos en su entorno competitivo, Tesla se ha centrado en posicionarse como un tipo diferente de empresa de automóviles basada en el desarrollo de una novedosa tecnología en materia de autos eléctricos, logrando separarse del modelo tradicional de los fabricantes de automóviles. A continuación, se presenta la tabla 1 con las principales aplicaciones novedosas.

Como una de las pioneras en el campo de los VE, Tesla ha desarrollado para trenes de potencia una tecnología global, integrada de propiedad eléctrica que constituye una ventaja competitiva por sus características innovadoras. Tanto el motor como la caja de cambios se fabrican en la empresa y todos los componentes de transmisión están integrado sinérgicamente para proporcionar un rendimiento superior en términos de costo y alcance (Hitt et al., 2014).

Por otro lado, la organización Tesla ha implementado una serie de estrategias novedosas como parte de su plan estratégico para mantener su liderazgo en el mercado de los vehículos eléctricos que cada vez es más competitivo. En la tabla 2, se aprecia un resumen de las principales estrategias y acciones que ha emprendido la mencionada empresa.

Tabla 1

Principales aplicaciones y características tecnológicas de Tesla Motor Inc

\begin{tabular}{lll}
\hline \multicolumn{1}{c}{ Innovación } & \multicolumn{1}{c}{ Características } & \multicolumn{1}{c}{ Valor agregado } \\
\hline $\begin{array}{l}\text { Tecnología de transmisión TES- } \\
\text { LA y sistema de alta eficiencia } \\
\text { eléctrica. }\end{array}$ & $\begin{array}{l}\text { La integración ofrece un sistema que es } \\
\text { mayor a la suma de sus partes con propiedad } \\
\text { intelectual profunda y protección de toda la } \\
\text { patente. }\end{array}$ & $\begin{array}{l}\text { Liderazgo del vehículo eléctrico en rango (millas máxi- } \\
\text { mas por carga individual) y en costo. } \\
\text { VE de cero emisiones de carbono. } \\
\text { Los VE viajan a mayor distancia con una carga que los } \\
\text { de la competencia. }\end{array}$ \\
\hline & $\begin{array}{l}\text { Permite para la recarga fuentes renovables } \\
\text { de electricidad, como la eólica y fotovoltáica. } \\
\text { Red Supercharger que proporciona una } \\
\text { recarga más rápida. }\end{array}$ & $\begin{array}{l}\text { Utilización de energías limpias. Y accesibles en diferen- } \\
\text { tes formatos. } \\
\text { Cargas más rápidas en comparación a la competencia. }\end{array}$ \\
\hline $\begin{array}{l}\text { Navegación con piloto automá- } \\
\text { tico - Navigate on Autopilot }\end{array}$ & $\begin{array}{l}\text { Sistema que ofrece una navegación más } \\
\text { fluida y eficiencia operativa. }\end{array}$ & $\begin{array}{l}\text { Viajes largos placenteros } \\
\text { Cambios de carril sugeridos automatizados. } \\
\text { Facilidad para seguir la guía de navegación cuando } \\
\text { viaja hacia una ruta desconocida. }\end{array}$ \\
\hline
\end{tabular}

Fuente: Elaboración propia adaptado de www.tesla.com/blog y de Hitt, Ireland y Hoskisson (2014) 
Tabla 2

Principales estrategias y acciones implementadas por Tesla Motors Inc.

\begin{tabular}{|c|c|}
\hline Estrategia comunicacional & $\begin{array}{l}\text { Aplica un sistema de comunicación basado en las TIC`s a todo lo largo de la organización que } \\
\text { abarca a los grupos de interés en el marco de la RSE y el compromiso ambiental. En el caso de los } \\
\text { clientes han implementado un programa de recomendaciones y mantienen de manera fluida la } \\
\text { comunicación donde capturan sus inquietudes y comentarios directamente desde sus centros de } \\
\text { exhibición y en línea. }\end{array}$ \\
\hline $\begin{array}{l}\text { Estrategia de Aventura conjuntay } \\
\text { asociaciones }\end{array}$ & $\begin{array}{l}\text { Panasonic (desarrollo de celdas de baterías de alta eficiencia con visión de futuro). } \\
\text { Daimler y Toyota (desarrollo de partes componentes de los VE). }\end{array}$ \\
\hline $\begin{array}{l}\text { Modelo innovador de ventas y } \\
\text { marketing }\end{array}$ & $\begin{array}{l}\text { Ventas directas para lo cual utiliza sus propios concesionarios donde suministra una atención per- } \\
\text { sonalizada a sus clientes y ofrece sus servicios de posventa. (salas de exhibición en Estados Unidos, } \\
\text { Canadá, Asia Pacífico y Europa con interacción para que el cliente explore y aprenda por sí mismo } \\
\text { acerca de la tecnología) } \\
\text { Impulso creciente de venta en línea para reducir infraestructura y transferir los ahorros al cliente } \\
\text { mediante mejores precios y beneficios con calidad certificada. } \\
\text { Ventas directas de partes de trenes de potencia patentados a otros fabricantes. } \\
\text { Variedad de modelos de VE para las diferentes necesidades y actualizaciones tecnológicas perma- } \\
\text { nentes. } \\
\text { Estrategia de Justo a Tiempo con autos hechos bajo pedidos y a la medida. }\end{array}$ \\
\hline Liderazgo y Cultura organizacional & $\begin{array}{l}\text { Equipo ejecutivo y de gestión conformado por especialistas de exitosa trayectoria que han guiado a } \\
\text { la compañía desde el diseño y la producción con visión estratégica. } \\
\text { Cultura emprendedora con énfasis en los valores de responsabilidad social, desarrollo sostenible y } \\
\text { visión creativa desde la elección de su personal para conformar el equipo Tesla. } \\
\text { Ambiente laboral abierto para fomentar la fuerza de trabajo creativa y cooperativa. }\end{array}$ \\
\hline
\end{tabular}

Fuente: Elaboración propia adaptado de www.tesla.com/blog y de Hitt. M., Ireland R. y Hoskisson R. (2014)

En la oportunidad de emitir el informe de impacto correspondiente al 15 de abril de 2019, donde se mide el impacto que los productos y operaciones tienen sobre el medio ambiente y las comunidades, la empresa Tesla manifiesta, que están trabajando arduamente para minimizar la huella de gases de efecto invernadero mediante la colocación cada vez más de sus VE con mejoras innovadoras, al tiempo que invierten en sus empleados y las comunidades donde operan. www.tesla.com/blog

\section{CONCLUSIONES}

1. Las organizaciones empresariales para lograr sus objetivos estrategicos y apalancar su crecimiento necesitan implementar la cultura de la innovación en todos los niveles jerarquicos de la estructura, considerando los cambios en las demandas de los mercados, la competividad y la responsabilidad social que corresponde según los impactos que genera, y los criterios para un desarrollo sostenible. En este sentido la empresa Tesla ha venido demostrando desde su creación que son una organización innovadora comprometida con el ambiente en el marco de la RS y que emplea de manera estartegica su plataforma tecnologica y de comunicación como factores clave de éxito.
2. Las estrategias comunicacionales de la organizaciones empresariales requieren ser formuladas e implementadas en el marco de la responsabilidad social empresarial atendiendo los requerimientos y necesidades de todos los grupos de interés. Para ello necesita apalancarse en las tecnologias de información y comunicación disponibles según los avances tecnológicos.

3. El sentido de responsabilidad social, nace en la conciencia y se fundamenta en principios morales y valores universales soportados por una ética organizacional. Es por ello que las organizaciones innovadoras deben invertir esfuerzos y recursos en la conformación de una cultura organizacional en concordancia con la innovación y la responsabilidad social.

4. La estrategia comunicacional aporta a las organizaciones innovadoras, canales y medios para posicionar su imagen y prestigio en las comunidades donde están inmersas, además de potenciar su producción y desarrollo de nuevos productos y servicios con sentido de responsabilidad social mediante un flujo permanente de conocimiento y experiencias, logrando un aprendizaje dinámico organizacional. 


\section{REFERENCIAS BIBLIOGRÁFICAS}

Foro Consultivo Científico y Tecnológico (FCCyT) A.C. y la OCDE (2012). La Estrategia de Innovación de la OCDE. Disponible en: http:// www.foroconsultivo.org.mx/libros_editados/ estrategia_innovacion_ocde.pdf .Consultada: 20/10/18.

Hitt. M., Black, J. y Porter, L. (2006). Administración. México: Editorial Pearson Educación 9ạ. edición.

Hitt. M., Ireland R. y Hoskisson, R. (2014). Administración estratégica. México: Editorial CENGAGE Learning. 11a․ edición.

Lescano, J., Valdéz, L., Lescano, L., Reyes, C., y Belaúnde, M. (2015). Manual del desarrollo sostenible. Lima, Perú: Macro EIRL.
Koontz, H., Weihrich, H., Cannice, M. (2012). Administración. Una perspectiva global y empresarial. México: Mc Graw Hill. 14ª . Edición.

Pérez, M. (2011). Gerencia Emergente en Empresas con Dinámicas de Cambio Organizacional. Revista Científica Digital del Centro de Investigación y Estudios Gerenciales. Disponible en: http://www.grupocieg.org/archivos_revista/2Esp.\%20Junio\%202011\%20(162-174)\%20 JIEGA\%20maura\%20p\%C3\%A9rez_Artículo_ id46.pdf. Consultada: 2/11/18.

Ramos, C. (2010). La universidad de servicio. Propuesta de un modelo interactivo entre instituciones socialmente responsable y el entorno socioproductivo. Editorial de la Universidad del Zulia (Ediluz).

Tesla Motors Inc. www.tesla.com/blog Consultada: 08/05/2019 